\title{
THE TREATMENT OF MULTIPLE LONG BONE FRACTURES
}

\author{
R Barker, SHO in Orthopaedics \\ Royal Lancaster Infirmary
}

\section{INTRODUCTION}

This article introduces a new development in the management of long bone fractures in trauma victims. Until recently multiple femoral fractures were treated by a combination of plating, nailing and long periods of bed rest and traction. There was no real way to fixate these difficult segmental fractures, particularly when associated with another proximal or distal fracture. The long periods of immobility had many complications, putting the patients at risk of significant morbidity and mortality from the moment of the injuries.

\section{CASE REPORT}

On the night of 4 November 1998 a young man was received in the accident and emergency department after a road traffic accident. He had lost control of his vehicle, which had plummeted 40 feet down an embankment. The ambulance men found him outside the Astra van, the window of which was smashed. It was unclear whether he was thrown through the windscreen or not. He later admitted to removing his safety belt to pick up some cigarettes from the passenger footwell.

On arrival in A\&E he was in considerable pain from his right leg. The paramedics had splinted this at the scene, suspecting multiple fractures. His minor injuries included lacerations to the face and fractures to the fourth and fifth metacarpals of the left hand. He sustained no intracranial or cervical spine injury.

Figure 1 shows the X-rays of his right leg on admission There is a comminuted fracture of the femur, a trochanteric
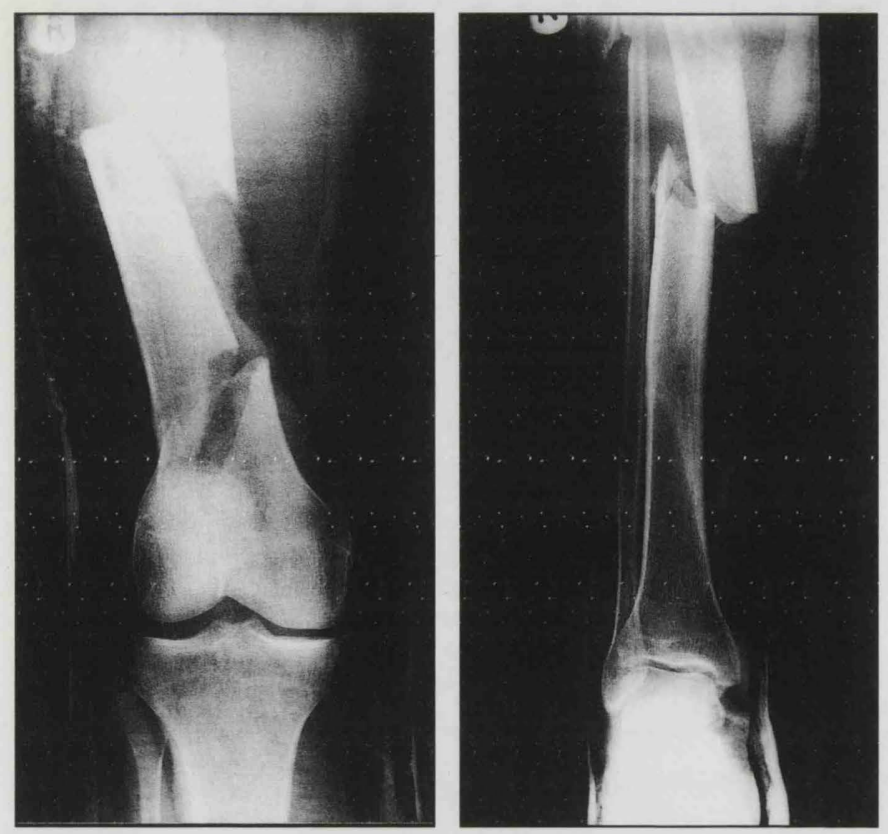

Figure 1 fracture, and at least two other segmental fractures, the lower being supracondylar. The tibia has sustained a comminuted segmental fracture in its upper third; there was a small skin wound at this site.

It was decided the patient should have urgent internal fixation, and ITU care as a multiply injured trauma victim. An ITU bed was available. Fortunately the necessary equipment was swiftly ordered, and available at RLI by $3.00 \mathrm{pm}$ the following day, some fourteen hours after the accident. The patient went from casualty straight to the ward, was transfused four units of blood overnight and taken to theatre the following afternoon.

A 'RECON' femoral nail was inserted. This is a metal rod placed within the medulla of the femur to provide internal fixation. The operation included pins to fix the trochanteric fracture, and distal locking screws to prevent rotation at the fracture sight. The segmental tibial fracture was also treated by an intermedullary nail in similar fashion. Prophylactic antibiotics were given on induction of the anaesthetic and continued for 48 hours, because this was an open fracture.

The post-operative X-rays are shown in Figure 2.

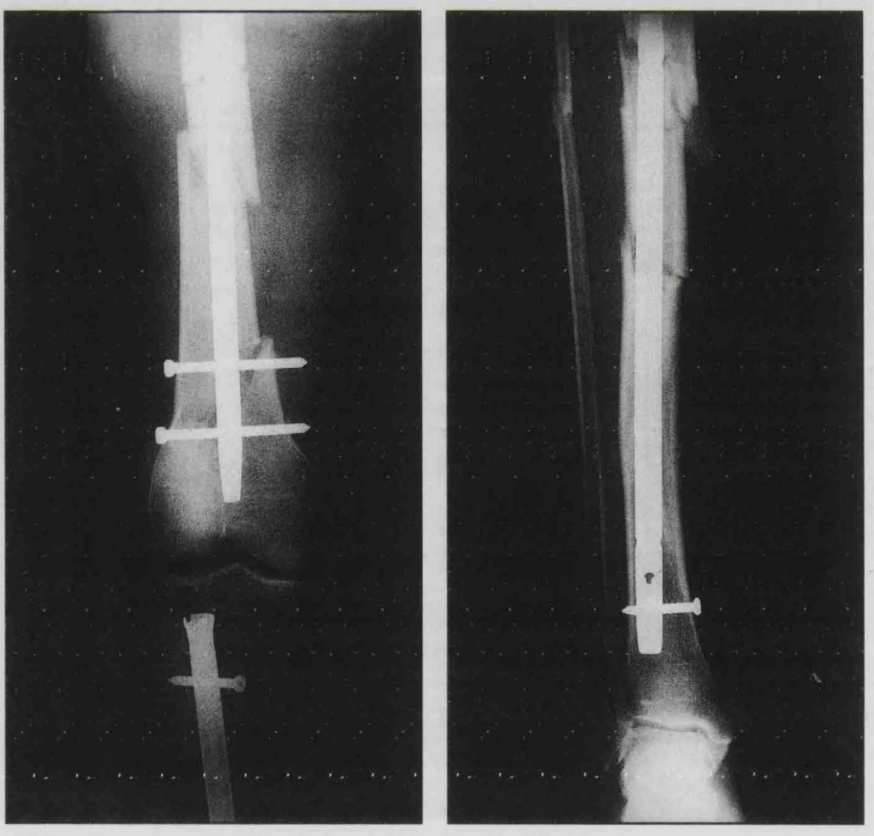

Figure 2

Post-operatively the patient returned to a general orthopaedic ward where he recovered remarkably well. He was alert, orientated and responding normally the following morning. His cardiovascular system was normal and stable, he was apyrexial, and his oxygen saturation was $98 \%$ on air. There were no signs of the common complications of traumatic long bone fractures. Non-weight-bearing 
mobilisation was started gradually. This usually lasts for six to eight weeks.

The possible complications of long bone fractures deserve some discussion, as they can be fatal to this group of young patients. Blood loss from the fracture site and infections in open injuries are common. Sepsis and disseminated intravascular coagulation can follow with disastrous consequences. Sepsis after trauma is more frequently fatal than sepsis in non-traumatic conditions because the systemic inflammatory response syndrome may result. By itself this can cause multiple organ failure and can compound previous injuries. A frequent and serious complication is the fat embolism syndrome. This is thought to occur to some degree in all long bone fractures, although often asymptomatic. Due to either direct marrow release or a generalised biochemical process, the fat droplets lodge in pulmonary capillaries and severe adult respiratory distress syndrome may ensue. Patients die in $50 \%$ of cases of symptomatic fat embolism due to refractory hypoxia.

Research has shown that immediate internal fixation of long bone fractures greatly reduces the incidence and severity of fat embolism, an almost universal and frequently fatal complication $^{(1)}$.

Internal fixation also allows the early mobilisation of the patients. This greatly reduces the incidence of all the complications associated with traction and the prolonged bed rest this entails. DVT, bed sores, hospital-acquired infections, boredom and a longer hospital stay were the major problems, and caused much morbidity and mortality.

The current method of choice for internal fixation of these difficult segmental fractures is the RECON intramedullary nailing system. It is well thought of and in widespread use throughout the country. We have used twelve of these nails at

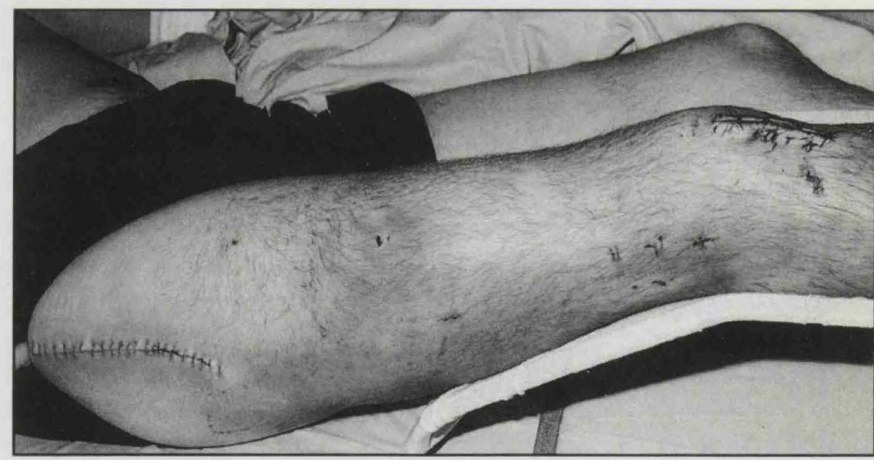

One day post-op

RLI over the last year for urgent cases in young trauma victims with great success. The orthopaedic team hopes soon to add this internal fixation system to our routine stock list, rather than the "order as required" system currently in place.

\section{CONCLUSION}

The appropriate and timely management of long bone fractures by rigid internal fixation using specialist devices is vital to prevent morbidity and mortality in young patients. These fractures should be fixed immediately after resuscitation or on the next day's trauma list at the latest.

\section{Acknowledgements}

Yvonne Dickinson, Medical Illustration; Mr H D Stewart, Consultant Orthopaedic Surgeon.

\section{REFERENCE}

1 Lhowe D, Hansen S, Immediate nailing of open fractures of the femoral shaft. JSJB 1988; 70(A):812-820 